\title{
Parental Marital Conflict and Growth in Adolescents' Externalizing Problems: the Role of Respiratory Sinus Arrhythmia
}

\author{
Yuan Peng ${ }^{1} \cdot$ Xiaohui Yang $^{1} \cdot$ Zhenhong Wang $^{1}$ \\ Accepted: 26 December 2020 / Published online: 9 February 2021 \\ (C) The Author(s) 2021
}

\begin{abstract}
Previous studies have examined the moderating effect of respiratory sinus arrhythmia (RSA) on the association between marital conflict and externalizing problems, however the findings were inconsistent. One possible reason is that the covariation of internalizing problems in externalizing problems. Therefore, the present study aimed to examine this issue. Participants were 332 Chinese adolescents (54.5\% boys) age from 13 to 15 years old. At T1, electrocardiogram monitoring was performed on adolescents during the resting state and stressor tasks (a speech task and a mental arithmetic task) to obtain RSA data. The Chinese version of the Achenbach Youth Self-Report-2001 (YSR-2001) and the Chinese version of the Children's Perception of Interparental Conflict scale were used to assess adolescents' externalizing and internalizing problems and their perception of marital conflict, respectively. Adolescents' problem behaviors were assessed again in the second and third waves of data collection, with a 1-year lag among each wave. The results revealed that the 3 - interactions of marital conflict $\times$ RSA reactivity in speech task $\times$ sex significantly predicted the trajectory of externalizing problems when controlling for internalizing problems from externalizing problems. Specifically, girls with greater RSA suppression to the speech task reported low and stable externalizing problems, however, boys with the same pattern were associated with slightly increased levels of externalizing problems. While, RSA augmentation to the speech task predicted the increase in externalizing problems among both girls and boys in high marital conflict families over time. However, this interaction effects were not significant when not partial out internalizing problems from externalizing problems. The findings highlight the importance of controlling for the covariation of internalizing problems when examining the interaction effects of person and environment on the development of adolescents' externalizing problems.
\end{abstract}

Keywords Externalizing problems $\cdot$ Marital conflict $\cdot$ Respiratory sinus arrhythmia $\cdot$ Adolescent $\cdot$ Controlling for internalizing problems

Externalizing problems (e.g., aggression and disruption) are common in children and adolescents and predict various maladaptive outcomes (e.g., Achenbach et al. 2016). The personenvironment interaction framework (e.g., Cicchetti 2008) proposes that environmental risk factors and individual vulnerability factors interactively contribute to children and adolescents' maladjustment such as externalizing problems. Among environmental risk factors, marital conflict has been regarded as a robust family stressor and is associated with externalizing problems (Cummings and Davies 2010; Grych and Fincham

Zhenhong Wang

wangzhenhong@snnu.edu.cn

1 School of Psychology, Shaanxi Provincial Key Research Center of Child Mental and Behavioral Health, Shaanxi Normal University, No.199, South Chang'an Road, Xi'an 710062, China
1990). Respiratory sinus arrhythmia (RSA) activity as an individual characteristic is considered a physiological marker of emotional self-regulation, and it has been found to be related to externalizing problems (Dietrich et al. 2007; Graziano and Derefink 2013). Moreover, several studies have found that RSA activity, including both baseline RSA and RSA reactivity, interacted with marital conflict to predict children's and preadolescents' externalizing problems (El-Sheikh et al. 2011; Katz and Gottman 1997; Obradović et al. 2011). However, some others have failed to find this moderating effect in children (Eisenberg et al. 2012; El-Sheikh and Whitson 2006; Whitson and El-Sheikh 2003).

One possible explanation for these inconsistent findings is that externalizing problems generally covary with internalizing problems (Achenbach et al. 2016; Keiley et al. 2003; Lilienfeld 2003), externalizing problems usually overlap with internalizing problems in other words. More importantly, 
RSA activities have different roles in the development of externalizing problems and internalizing problems (Graziano and Derefink 2013). It is possible that the moderating effect of RSA activity on the association between marital conflict and externalizing problems might be affected by the covariance of internalizing problems. However, few existing studies control for internalizing problems when examining the associations among marital conflict, RSA activity, and externalizing problems. Thus, the present study aimed to explore this issue among a sample of Chinese adolescents.

\section{Marital Conflict and Externalizing Problems}

Marital conflict is defined as any difference, disagreement, or argument regarding an issue of family life, and it includes all kinds of psychological and physical conflicts between couples (Cummings and Davies 2010). Marital conflict can be considered a chronic stressor and serves as a developmental risk factor for children and adolescents. Several influential models elucidate the potential mechanisms and processes regarding how marital conflict affects child functioning. In the cognitivecontextual framework (Grych and Fincham 1990), children and adolescents' distorted social cognitive and behavioral patterns, such as dysfunctional attributions and negative self-representation, are proposed to mediate the link between parental conflict and their psychopathology. The emotional security hypothesis (Cummings and Davies 2010) proposes that marital conflict might affect children and adolescents' emotional security and emotional regulation ability, further increasing the risk of problem behaviors such as externalizing problems. In addition to undermining cognitive, behavioral and emotional aspects, marital conflict also has a negative effect on children and adolescents' adaptation by compromising physiological function. Previous studies have suggested that chronic physiological and emotional arousal elicited by frequent marital conflict may result in allostatic load and stress vulnerability to the environment; such a process could be an important mechanism in the development of psychopathology (El-Sheikh and Erath 2011). Large bodies of cross-sectional and longitudinal studies have suggested that marital conflict is associated with an increased risk of children and adolescents' maladjustment, including externalizing problems (Cummings and Davies 2010; Grych and Fincham 2001; Tu et al. 2016).

\section{RSA and Externalizing Problems}

Based on theoretical and empirical work (Beauchaine et al. 2007; Porges 1995, 2007), individual RSA activity, which is conceptualized as a physiological index of emotional and behavioral regulation, plays an important role in individual differences in externalizing problems. RSA refers to rhythmic fluctuations in an individual's heart rate corresponding to the natural respiratory cycle. RSA during the resting state (baseline RSA) and changes in RSA in response to environmental challenges (RSA reactivity) are both processes of parasympathetic nervous system (PNS) functioning in the heart via the vagus nerve (Porges 1995, 2007). Baseline RSA reflects the individual's ability to maintain homeostasis and a calm state, and a high baseline RSA is proposed to be associated with adaptive functioning (Calkins et al. 2007; Porges 1995, 2007). Generally, RSA suppression (decreased RSA) during stress or a high-challenge context supports the ability to modulate arousal and to facilitate the mobilization of physiological resources to flexibly adapt to environmental demands (Calkins et al. 2007; Graziano and Derefink 2013; Porges 1995, 2007). However, in a low-risk or low-challenge context, such a decrease in RSA is considered unnecessary and may not represent adaptive physiological regulation (Davis et al. 2016; Hastings et al. 2008). In contrast, RSA augmentation (increased RSA) during stress may reflect deficits in the capacity to flexibly respond to demanding environments and may represent vulnerability (Graziano and Derefink 2013; Porges 1995, 2007; Mcquade and Breaux 2017). However, evidence regarding direct links between baseline RSA/RSA reactivity and externalizing problems among children is inconsistent. It has been suggested that RSA activity only confers risk for externalizing problems under certain conditions. As previous studies suggested, the bivariate relation between RSA activity and externalizing problems may be moderated by other factors, such as environmental factors (El-Sheikh and Erath 2011; Graziano and Derefink 2013).

\section{Marital Conflict, RSA and Externalizing Problems}

The person-environment interaction framework proposes that an individual's maladjustment is not determined alone by environmental factors or individual characteristics, and both environmental factors and individual characteristics interactively contribute to an individual's maladjustment (e.g., Cicchetti 2008). Furthermore, there are different perspectives regarding the nature of the interaction between environmental factors and individual characteristics when predicting developmental outcomes. The diathesis-stress model emphasizes that individuals who possess vulnerability characteristics are at higher risk of psychopathology in the context of environmental stressors (Monroe and Simons 1991). The differential susceptibility model (Belsky and Pluess 2009) proposes that individuals with susceptible characteristics are more sensitive to both negative and positive environments and thus are prone to maladjustment or positive adjustment. Based on these theories, empirical studies have explored the extent to which marital conflict and RSA activity interact to predict children's 
externalizing problems; however, the findings are mixed. Some studies have found that baseline RSA moderated the association between marital conflict and children's externalizing problems (El-Sheikh et al. 2001; El-Sheikh et al. 2011; Katz and Gottman 1997). For example, El-Sheikh et al. (2001) found that high baseline RSA buffered 8- to 12-year-old children against the negative effect of marital conflict, whereas low baseline RSA and marital conflict interactively predicted more externalizing problems. Others have failed to find this moderating effect in children (Eisenberg et al. 2012; ElSheikh and Whitson 2006; Whitson and El-Sheikh 2003). Similarly, some studies have found that RSA reactivity to stress tasks moderated the association between marital conflict and children's externalizing problems (El-Sheikh et al. 2001; El-Sheikh et al. 2011; Obradović et al. 2011). For example, Obradović et al. (2011) found that 4- to 6-year-old children who showed greater RSA suppression in response to interpersonal task were less affected by marital conflict, while children who showed RSA augmentation exhibited more externalizing problems in the context of marital conflict. Other studies have shown that RSA reactivity to stress tasks such as listening to the mock adult argument task did not moderate the association between marital conflict and children's externalizing problems (El-Sheikh and Whitson 2006; Whitson and El-Sheikh 2003).

Taken together, the findings regarding the interaction effect of RSA activity and marital conflict on externalizing problems were inconsistent. There may be several reasons. One probable reason lies in the comorbidity or covariation of externalizing and internalizing problems. A large body of literature has demonstrated that externalizing and internalizing problems are closely related and often involve covariation and codevelopment during childhood and adolescence (Achenbach et al. 2016; Angold and Costello 1993). According to the bifactor model (Chen et al. 2006; Wang et al. 2016 and Wang, Eisenberg, Valiente, \& Spinrad, 2016), the externalizing problems and the mixed profile of externalizing problems (i.e. including covariation of externalizing and internalizing problems) may have different antecedents, correlates, developmental pathway, and outcomes. Several studies have found that different patterns of externalizing problems are related to differential patterns of physiological reactivity, including RSA reactivity (e.g., Calkins et al. 2007). For example, Calkins et al. (2007) found that children at risk for a mixed profile of externalizing problems displayed the greatest RSA suppression in response to environmental challenges, while children with a pure externalizing problem displayed the least RSA suppression in response to environmental challenges. Thus, the contribution of RSA activity to externalizing problems may be affected by the covariance of externalizing and internalizing problems. In samples of children and adolescents, a lower baseline RSA was reported to be positively (Bosch et al. 2009; Dietrich et al. 2011), negatively (Blood et al. 2015; Pang and Beauchaine 2013), or nonsignificantly (Koenig et al. 2016) related to internalizing problems. Meanwhile, a lower baseline RSA was reported to be positively (Beauchaine et al. 2007) or negatively (Dietrich et al. 2007; Xu et al. 2014) related to externalizing problems. Similarly, either greater RSA suppression (Graziano and Derefink 2013) or RSA augmentation(Thayer and Lane 2000) in response to stressors has been found to be linked with more internalizing problems and RSA augmentation has been found to be linked with more externalizing problems (Davis et al. 2016; Mcquade and Breaux 2017). Considering that the direction of the associations between RSA activity (baseline RSA and RSA reactivity) and internalizing and externalizing problems may be different, the role of RSA activity in the relationship between marital conflict and externalizing problems might be confused and even offset by the covariance of externalizing and internalizing problems. Therefore, as previous studies suggested (Achenbach et al. 2016; Keiley et al. 2003; Lilienfeld 2003), more precise conclusions could have been drawn regarding the moderating effect of RSA activity (baseline RSA and RSA reactivity) on the association between marital conflict and externalizing problems when controlling for internalizing problems.

The second reason of the inconsistent finding is partly due to sex differences in associations among marital conflict, RSA, and externalizing problems. For example, some studies have found that marital conflict predicted increases in externalizing problems over time, especially among boys with lower baseline RSA or less RSA suppression (El-Sheikh et al. 2001; El-Sheikh and Erath 2011). Thus, the present study also aimed to examine whether the associations between RSA activity, marital conflict and externalizing problems when controlling for internalizing problems differ by sex. This may further delineate sex-specific pathways underlying such associations.

Lastly, as previous studies suggested, the types of stressor tasks used to evoke RSA reactivity, might affect the moderating effect of RSA reactivity on the relationship between family environment and psychological adaptation among children and adolescents (Cui et al. 2015; Obradović et al. 2011). Consequently, the present study measured RSA reactivity during speech task and mental arithmetic task to further clarify the moderating effect of RSA reactivity on the association between marital conflict and externalizing problems. The speech task and mental arithmetic task are widely used to obtain stress responses (Dickerson and Kemeny 2004; Lü et al. 2016). Due to the task characterized with social-evaluative threat and uncontrollability, the speech task is known as a stressful situation or high-challenge task (Dickerson and Kemeny 2004; Palatini et al. 2011). In this context, greater RSA suppression, which reflects physiological arousal and the potential ability to cope effectively in response to social stressors, may represent an adaptive physiological response and thus may moderate the association between marital conflict and adolescents' externalizing problems when controlling for internalizing problems. The mental arithmetic task, which is classified as one 
of the cognitive tasks, is designed to evoke a stress response by testing participants' cognitive function. Because this task has been suggested to represent a low-challenge context (Dickerson and Kemeny 2004; Palatini et al. 2011), greater RSA suppression in response to such task may be unnecessary and may not fully reflect individual's capacity to modulate physiological and emotional states in coping with stress such as marital conflict. Thus, greater RSA suppression in response to mental arithmetic task may not moderate the association between marital conflict and adolescents' externalizing problems when controlling for internalizing problems.

In addition, most studies that examined the effect of the interaction between RSA activity and marital conflict on externalizing problems were conducted in children. However, few studies have examined the effect of this interaction among early adolescents. Previous studies have suggested that early adolescence is regarded as a critical period during which family relationships, individuals' psychological states and stress physiology systems, including the PNS, undergo marked developmental changes as well as a period of increased vulnerability (McCormick et al. 2010). Hence, it is important to examine the association among marital conflict, RSA activity and the development of externalizing problems among early adolescents.

\section{The Present Study}

In summary, the main aim of the present study was to examine the interaction of RSA activity and marital conflict on the development of adolescents' externalizing problems when controlling for internalizing problems. The present study used a longitudinal design with three waves of data collection among Chinese adolescents. Latent growth curve model (LGCM) was used in the present study to explore the trajectory of externalizing problems. This method allows for the identification of levels of externalizing problems, changes in externalizing problems over time and how marital conflict and RSA activity interact to predict the trajectory of externalizing problems (Martinstorey et al. 2018; Singer and Willett 2003).

Considering that the role of marital conflict and RSA activity (including baseline RSA and RSA reactivity) might be confused or offset by the covariance of externalizing and internalizing problems, we hypothesized that the interaction of marital conflict and RSA activity on the development of adolescents' externalizing problems might vary with whether internalizing problems are controlled for. Based on previous study (Calkins et al. 2007), we hypothesized that when controlling for internalizing problems (i.e. removing the impact of the covariance of internalizing problems), marital conflict and RSA activity (baseline RSA and RSA reactivity) could predict the development of adolescents' externalizing problems. Whereas, when not controlling for internalizing problems, the interaction of marital conflict and RSA activity on externalizing problems were not significant.
Furthermore, adolescents' sex and the type of challenge task might moderate the associations among marital conflict, RSA activity, and adolescents' externalizing problems. We anticipated that these associations would be stronger among boys than girls on the basis of existing empirical evidence (El-Sheikh et al. 2001; El-Sheikh and Erath 2011). Moreover, we anticipated RSA reactivity in response to speech task other than mental arithmetic task may moderate the association between marital conflict and adolescents' externalizing problems when controlling for internalizing problems.

\section{Method}

\section{Participants}

The data were collected as part of a larger project designed to explore the effects of family environment and adolescent characteristics on the development of adolescents' problem behaviors. The longitudinal design consisted of three annual measurement occasions, with retention rates of $90 \%$ and $92 \%$. We recruited adolescents when they were in grade seven (the first year in junior high school) from one ordinary junior high school in a city of northwestern China via flyers. The inclusion criteria were as follows: no diagnosis of a pervasive developmental disorder or chronic illness, and parents living together for three or more years at recruitment. During the first assessment (T1), 332 junior high school students (181 boys, $M_{\text {age }}=13.7$ years, standard deviation $(S D)=0.8)$ participated in the study. During the second assessment (T2) and third assessment (T3), 298 (149 boys, $M_{\text {age }}=14.8$ years, $\left.S D=0.5\right)$ and 274 (143 boys, $M=15.6$ years, $S D=0.6)$ adolescents again reported their externalizing and internalizing problems. Almost all participants (95\%) were of Chinese Han ethnicity, and all participants spoke mandarin. All the participants' parents were not divorced and lived together with the participated adolescent.

\section{Procedure}

T1 The present study was approved by the XXX university research ethics committee. Written informed consent was obtained from all participants and their parents prior to data collection, and the participants were informed of the nature of the study and their right to withdraw from the investigation at any time. The participants came to the laboratory at the scheduled appointment times (ranging from 2:00 p.m. to 6:00 p.m. for four weeks). Before the formal test was conducted, detailed instructions were provided to ensure that the participants clearly understood the experimental procedures. To eliminate the risk of any exogenous effects on the physiological measures, all participants were instructed to refrain from performing physical exercise or consuming any caffeine or alcohol for two hours before the experiment. After completing 
the questionnaires, the adolescents participated in the laboratory tasks during which their physiological data were measured. First, electrocardiogram (ECG) recording electrodes were attached to the participants. The participants were then given $10 \mathrm{~min}$ to acclimate to the laboratory and to relax. Then, the formal physiological experiment began, with all instructions for the experimental procedure being simultaneously presented on a computer screen.

The entire physiological experiment included five phases (see Fig. 1). First, the participants were instructed to look at a neutral picture shown on the computer screen (a picture of a cup selected from the International Affective Picture System, IAPS; Lang et al. 2005) to calculate their 5-min baseline RSA values. Then, the participants were instructed to complete a mental arithmetic task and to perform a speech task to induce stress responses. Between the two tasks, the participants rested for $5 \mathrm{~min}$, during which they were instructed to relax and look at neutral pictures. After each phase, the participants were asked to rate their subjective emotional experience (SEE), including nervousness and anxiety, on two 5-point scales ranging from 0 ("relaxed") to 4 ("nervous" or "anxious"). The sequences of the two stressor tasks were balanced to eliminate the impact of order effects.

T2 and T3 The adolescents were invited back to the research laboratory at $\mathrm{T} 2$ and $\mathrm{T} 3$, and they completed the questionnaire again. All participants were compensated $¥ 60$ (approximately $\$ 10$ ) for their participation.

\section{Stress Tasks}

\section{Mental Arithmetic Task}

The participants were informed that their mathematical skills would be evaluated based on their performance in the task, in which they were asked to repeatedly subtract 13 from fourdigit numbers presented on a computer (Diamond et al. 2012). The participants were asked to state the results of their calculation before the correct answer was displayed every $4.5 \mathrm{~s}$ on the monitor after the beep. To avoid social-evaluative threat, the two research assistants who were present with them left the experiment room when the participants performed the mental arithmetic task.

\section{Speech Task}

The participants were told to give a speech for a mock class leader election, and two research assistants served as live interviewers. The participants were provided with the following instructions (originally given in Chinese): "You will deliver a speech for a class leader election, for which you have $2 \mathrm{~min}$ to prepare; then, you will have $5 \mathrm{~min}$ to state the type of position you are running for, as well as the reasons you are qualified for this position. Your performance will be evaluated by the research assistants in terms of overall content, clarity, and delivery." After the preparation phase, the participants delivered their 5 min speech to the two assistants. Whenever the participants paused for more than $10 \mathrm{~s}$, they were prompted to continue. The validity of the speech task has been verified in previous studies (Lü et al. 2016).

\section{Measures}

\section{Marital Conflict (Measured at T1)}

The conflict characteristic subscale of the Chinese version of the Children's Perception of Interparental Conflict scale (Grych and Fincham 1990; Chi and Xin 2003) was used to measure marital conflict. This 19-item subscale includes three dimensions: conflict frequency (six items; e.g., "I often see my parents arguing"), conflict intensity (seven items; e.g., "When my parents have an argument, they yell a lot"), and conflict resolution (six items; e.g., "Even after my parents stop arguing, they stay mad at each other"). The three dimensions are summed to create a single overall measure of marital conflict, with higher scores indicating greater marital conflict. Responses are given using a 5-point scale ranging from 1 ("never") to 5 ("always"). The scale was found to have adequate internal consistency reliability $(\alpha=0.81)$.

\section{Externalizing Problems and Internalizing Problems (Measured at $\mathrm{T} 1, \mathrm{~T} 2$ and $\mathrm{T} 3$ )}

The Chinese version of the Achenbach Youth Self-Report-2001 was used to assess adolescents' externalizing and internalizing problems (Achenbach and Rescorla 2001; Wang et al. 2016 and Wang, Eisenberg, Valiente, \& Spinrad, 2016). The 33-item externalizing problem subscale measures aggression and

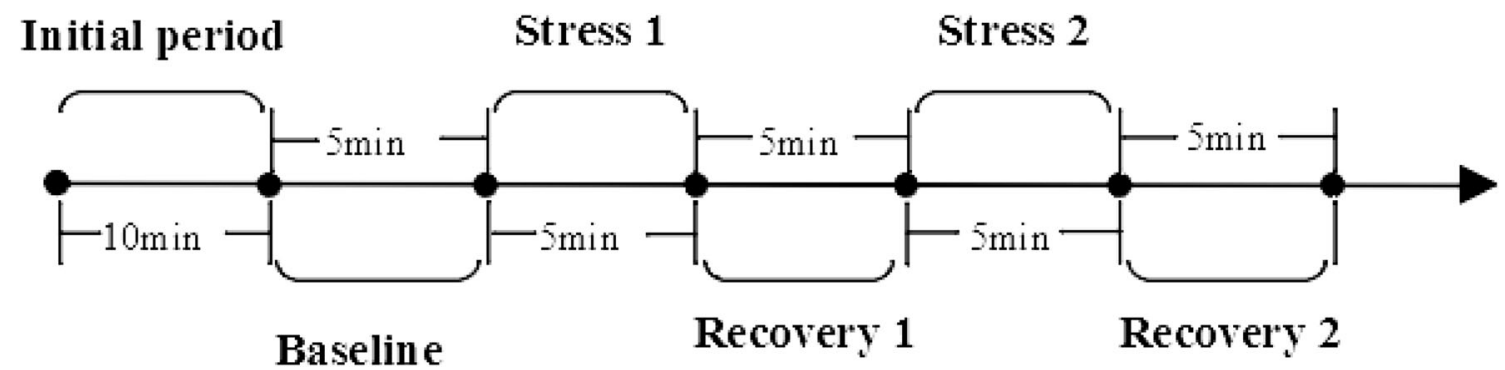

Fig. 1 Time-line of experimental session 
disruption. The 27-item internalizing problem subscale measures withdrawal/depression, anxiety/depression, and somatic complaints. The item scores are summed to create overall measures of externalizing and internalizing problems, with higher scores indicating more externalizing and internalizing problems. Responses are provided using a 3-point scale ranging from 0 ("not true") to 2 ("very true or often true"). The externalizing and internalizing problem subscale had adequate internal consistency reliability at T1, T2 and T3 ( $\alpha=0.82$ and $0.84 ; \alpha=0.80$ and $0.82 ; \alpha=0.79$ and 0.81 , respectively).

\section{Physiological Data (Measured at T1)}

The ECG data were continuously recorded using the SOMNO touch $^{\text {TM }}$ RESP (SOMNO medics, Germany), with a sampling rate of $256 \mathrm{~Hz}$. The ECG data were then transferred into DOMINO light software 1.4.0 (SOMNO medics, Germany) for the automatic detection of artifacts. Based on the smoothness prior approach, the $\mathrm{R}-\mathrm{R}$ intervals were resampled at $4 \mathrm{~Hz}$ and detrended (Tarvainen et al. 2002). Then, RSA was quantified via a high-frequency interbeat-interval power spectrum (0.22-0.40 Hz for adolescents aged 13 years and $0.20-$ $0.40 \mathrm{~Hz}$ for adolescents aged 14; Shader et al. 2018) corresponding to the respiratory cycle, and the values were natural$\log$-transformed $(\mathrm{ln})$ to fit the assumption of linear analyses, yielding $\ln$ units $\left(\mathrm{ms}^{2}\right)$. During each task, RSA was calculated by subtracting the average RSA during the baseline period from the average RSA during the specified task. Thus, negative scores correspond to significant decreases from baseline (RSA suppression), and positive scores correspond to significant increases from baseline (RSA augmentation).

\section{Missing Data and Imputation}

The adolescents with missing data were not different from the other adolescents in the sample in terms of any research variable ( $t s<1.23$, ps $>0.20)$. We used multiple imputation (MI; Schafer and Graham 2002) procedures available in Mplus version 7.4 to address the missing data. The MI procedures used the study variables and auxiliary variables to create 20 imputed datasets. After the MI procedures, each missing value was replaced by a mean of the 20 imputed values for that participant. Finally, datasets from the participants were used for statistical analyses.

\section{Data Analysis Plan}

Following guidelines established for LGCM (Singer and Willett 2003), we fit unconditional linear growth models at $\mathrm{T} 1, \mathrm{~T} 2$, and $\mathrm{T} 3$ as the observed variables. The latent intercept is interpreted as the mean value of externalizing at T1, and the latent slope regression parameters were fixed at 0,1 , and 2 . Because of having only 3 time points, a quadratic model was not tested (Singer and Willett 2003).
After determining that significant individual differences existed in the growth parameters of externalizing problems, predictors were added one at a time to the unconditional models to examine the interaction between marital conflict and RSA activity. We added the main effect, two-way interactions, and three-way interactions to the growth model. Moreover, we entered internalizing problems as covariates into the model. Because internalizing problems were measured at each time point, they can be considered timevarying covariates. The fit of the growth models was assessed using the chi-square statistic, the comparative fit index (CFI), the Tucker-Lewis index (TLI), and the root mean square error of approximation (RMSEA). Hu and Bentler (1999) suggested that a well-fitting model has a CFI or TLI greater than 0.95 and an RMSEA less than 0.06. The Johnson-Neyman technique (Bauer and Curran 2005) was used to plot a simple effect graph to better understand the interaction effects on the intercept of externalizing problems. Moreover, the interactions on the slope of the externalizing problems can further be illustrated by using the well-established procedure of constructing prototypical plots (Singer and Willett 2003).

\section{Results}

\section{Preliminary Analyses}

The means and standard deviations of the variables are provided in Table 1. ${ }^{1}$ During the speech task, ${ }^{2} 110$ (33.1\%) adolescents displayed RSA augmentation, and 222 (66.9\%) adolescents displayed RSA suppression. During the mental arithmetic task, ${ }^{3} 130(39.2 \%)$ adolescents displayed RSA

\footnotetext{
${ }^{1}$ T-tests revealed significant sex differences in externalizing problems and internalizing problems at $\mathrm{T} 3$, boys had significantly higher scores on the externalizing problems compared to girls, $t(330)=3.01, p<0.01$, and boys had significantly lower scores on the internalizing problems compared to girls, $t(330)=2.26, p<0.05$.

${ }^{2}$ Regarding the speech task, the repeated measures ANOVA also showed that the main effects of social stressors were significant for the SEE $(F[1.84$, $\left.6.6 .41]=160.91, p<0.001, \eta_{p}^{2}=0.33\right)$ and $\operatorname{RSA}(F[1.74,541.12]=90.86$, $p<0.001, \eta_{p}^{2}=0.23$ ). Pairwise comparisons (Bonferroni) showed that the RSA score in the challenge task was significantly lower than in the baseline and recovery periods $(p<0.001)$. There was no significant difference in the SEE and RSA scores between baseline and recovery $(p>0.05)$. These findings showed that using a speech task was effective in inducing changes in individuals' subjective and RSA reactivity.

${ }^{3}$ Regarding the mental arithmetic task, the repeated measures ANOVAs showed that the main effects of cognitive stressors were significant for the $\operatorname{SEE}\left(F[1.99,655.32]=75.91, p<0.001, \eta_{p}^{2}=0.19\right)$ and RSA $(F[1.739$, $535.04]=53.76, p<0.001, \eta_{p}^{2}=0.15$ ). Pairwise comparisons (Bonferroni) showed that the SEE and RSA scores in the challenge task were significantly lower than in the baseline and recovery periods $(p<0.001)$. There was no significant difference in the SEE and RSA scores between baseline and recovery $(p>0.05)$. These results showed that using a mathematical challenge task was effective in inducing changes in individuals' subjective and RSA reactivity.
} 
Table 1 Descriptive statistics and correlations among variables

\begin{tabular}{|c|c|c|c|c|c|c|c|c|c|c|c|}
\hline & 1 & 2 & 3 & 4 & 5 & 6 & 7 & 8 & 9 & 10 & 11 \\
\hline $1 \mathrm{Sex}$ & 1 & & & & & & & & & & \\
\hline 2 Marital conflict & -0.04 & 1 & & & & & & & & & \\
\hline 3 Baseline RSA & -0.04 & 0.01 & 1 & & & & & & & & \\
\hline 4 RSAR (mental arithmetic task) & 0.05 & -0.07 & $-0.41 * *$ & 1 & & & & & & & \\
\hline 5 RSAR (speech task ) & 0.04 & -0.05 & $-0.42 * *$ & $0.62 * *$ & 1 & & & & & & \\
\hline 6 T1 Internalizing problem & -0.07 & $0.22 * *$ & 0.05 & 0.05 & -0.01 & 1 & & & & & \\
\hline 7 T2 Internalizing problem & -0.09 & $0.17 * *$ & -0.05 & 0.08 & 0.04 & $0.46^{* *}$ & 1 & & & & \\
\hline 8 T3 Internalizing problem & $-0.15^{*}$ & $0.26 * *$ & 0.01 & 0.04 & 0.03 & $0.47 * *$ & $0.50 * *$ & 1 & & & \\
\hline 9 T1 Externalizing problem & 0.08 & $0.25 * *$ & 0.05 & 0.08 & 0.04 & $0.74 * *$ & $0.34 * *$ & $0.32 * *$ & 1 & & \\
\hline $10 \mathrm{~T} 2$ Externalizing problem & 0.10 & $0.28 * *$ & -0.09 & $0.16^{* *}$ & $0.17 * *$ & $0.36^{* *}$ & $0.63 * *$ & $0.46^{* *}$ & $0.42 * *$ & 1 & \\
\hline $11 \mathrm{~T} 3$ Externalizing problem & $0.18 * *$ & $0.28 * *$ & -0.07 & $0.13 *$ & $0.17 * *$ & $0.28 * *$ & $0.31 * *$ & $0.66^{* *}$ & $0.39 * *$ & $0.58 * *$ & 1 \\
\hline Mean & - & 40.21 & 6.33 & -0.10 & -0.11 & 12.01 & 14.35 & 15.66 & 9.15 & 9.70 & 11.40 \\
\hline$S D$ & - & 10.75 & 1.14 & 0.56 & 0.55 & 8.40 & 9.38 & 9.92 & 7.03 & 7.76 & 6.84 \\
\hline
\end{tabular}

$* p<0.05, * * p<0.01, * * * p<0.001$

augmentation, and 202 (60.8\%) adolescents displayed RSA suppression.

The unconditional externalizing problem model fit the data well $(\chi 2(d f)=1.42(1), \mathrm{CFI}=0.997, \mathrm{TLI}=0.991, \mathrm{RMSEA}=$ $0.037)$. The means for both the intercept and slope were significant (intercept $M=9.03, S E=0.39, p<0.001$; slope $M=$ $1.28, S E=0.23, p<0.001)$, indicating that, on average, externalizing problems increased over time. The correlation between the slope and the intercept was not significant $(r=$ $0.04, p>0.05)$. The variances of the intercept $\left(S^{2}=28.00\right.$, $S E=.6 .43, p<0.001)$ and slope $\left(S^{2}=8.70, S E=2.87, p<0\right.$ $.01)$ were significant.

\section{Conditional Models}

\section{Examinations of Interactions between RSA Reactivity (Speech Task) and Marital Conflict}

The parameter estimates for the final fitted model fit the data well $(\chi 2(d f)=17.31(14)$, CFI $=0.995$, TLI $=0.989$, RMSEA $=0.023$ ). As shown in Table 2 and Fig. 2, the two-way interaction between RSA reactivity (speech task) and marital conflict was a significant predictor of the intercept of externalizing problems when controlling for baseline RSA and internalizing problems $(B=0.52, S E=0.25, p<0.05)$. The JohnsonNeyman technique revealed that (see Fig. 3) there were significant positive relationships between marital conflict and adolescents' externalizing problems when RSA reactivity was greater than $-0.48(-0.68 \mathrm{Z})$.

The three-way interaction between RSA reactivity (speech task), marital conflict and sex was a significant predictor of the slope of externalizing problems when controlling for baseline RSA and internalizing problems $(B=$ $-0.32, S E=0.16, p<0.05)$.

\section{Prototypical Plots}

Figure $4 a-b$ present prototypical plots of growth in externalizing problems for girls and boys exposed to lower and higher levels of marital conflict. The two-way interaction significantly predicted the intercept, and the three-way interaction significantly predicted the slope. For girls and boys experiencing higher marital conflict, those exhibiting RSA augmentations have the highest levels of externalizing problems at each time point. For girls in high-conflict homes, those exhibiting RSA suppression showed the lowest and stable externalizing problems $(p>0.05)$. For boys in low-conflict homes, those exhibiting RSA suppression showed a slight increase $(p<0.05)$, while for boys in high-conflict homes, those exhibiting RSA suppression showed a significant increase over time $(p<0.001)$.

As shown in Table 3, the two-way interaction between RSA reactivity (speech task) and marital conflict and the three-way interaction between RSA reactivity (speech task), marital conflict and sex did not significantly predict either the intercept or the slope of externalizing problems when not controlling for internalizing problems $(p s>0.05)$.

\section{Examinations of the Interactions between Baseline RSA/RSA Reactivity (Mental Arithmetic Task) and Marital Conflict}

As shown in Table 2, two interactions between baseline RSA or RSA reactivity to the mental arithmetic task and marital conflict and the three-way interactions between baseline RSA or RSA reactivity to the mental arithmetic task, marital conflict and adolescents' sex did not significantly predict growth in externalizing problems when controlling for internalizing problems ( $p s>0.05)$. 
Table 2 Estimates for predictors of externalizing problems trajectory (control for internalizing problems)

Model 1

Main effects

Sex

Marital conflict

Baseline RSA

2-way interactions

Marital conflict $\times$ Baseline RSA

Sex $\times$ Marital conflict

Sex $\times$ Baseline RSA

3-way interaction

Marital conflict $\times$ Baseline RSA $\times$ Sex

Residual variance

$R^{2}$

Model 2

Baseline RSA

Main effects

Sex

Marital conflict

RSA reactivity (speech task)

2-way interactions

Marital conflict $\times$ RSA reactivity (speech task)

Sex $\times$ Marital conflict

Sex $\times$ RSA reactivity (speech task)

3-way interaction

Marital conflict $\times$ RSA reactivity (speech task) $\times$ Sex

Residual variance

$R^{2}$

Model 3

Baseline RSA

Main effects

Sex

Marital conflict

RSA reactivity (mental arithmetic task)

2-way interactions

Marital conflict $\times$ RSA reactivity (mental arithmetic task)

Sex $\times$ Marital conflict

Sex $\times$ RSA reactivity (mental arithmetic task)

3-way interaction

Marital conflict $\times$ RSA reactivity (mental arithmetic task) $\times$ Sex

Residual variance

$R^{2}$
Intercept $(M=1.66, S E=0.44)$
$B \quad S E$

0.91

0.93

$-0.03$

0.25

0.26

0.26

$3.59 * * *$

$3.52 * * *$

$-0.09$

$-0.13$

0.52

$-0.31$

0.25

$-0.51$

$2.03 *$

$-1.23$

$-0.28$

0.24

$-1.15$

5.69

$25.8 \%$

Intercept $(M=1.75, S E=0.44)$

$B \quad S E \quad t$

0.31

0.28

1.10

0.90

0.25

$3.62 * * *$

0.97

0.63

0.26

$3.70 * * *$

0.28

$2.25 *$

0.52

0.53

0.36

\subsection{5}

\section{$2.04 *$}

$2.14 *$

1.10

0.28

0.25

1.17

4.21

$39.6 \%$

Intercept $(M=1.72, S E=0.44)$

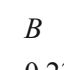

0.23

0.89

0.94

0.47

0.42

0.51

0.24

0.54

4.89

$33.2 \%$
Slope $(M=1.63, S E=0.35)$

$B \quad S E \quad t$

$\begin{array}{lll}0.47 & 0.16 & 2.85 * * \\ 0.06 & 0.19 & 0.31 \\ -0.25 & 0.17 & -1.50\end{array}$

$\begin{array}{lll}-0.04 & 0.17 & -0.36\end{array}$

$\begin{array}{lll}-0.03 & 0.17 & -0.24\end{array}$

$\begin{array}{lll}0.13 & 0.16 & 0.81\end{array}$

$\begin{array}{lll}0.21 & 0.16 & 1.36\end{array}$

1.82

$18.8 \%$

Slope $(M=1.60, S E=0.34)$

$B \quad S E \quad t$

$\begin{array}{lll}-0.17 & 0.19 & -0.89\end{array}$

$\begin{array}{lll}0.45 & 0.17 & 2.73 * *\end{array}$

$\begin{array}{lll}0.08 & 0.19 & 0.43\end{array}$

$\begin{array}{lll}0.25 & 0.19 & 1.36\end{array}$

$\begin{array}{lll}-0.06 & 0.16 & -0.34\end{array}$

$\begin{array}{lll}-0.02 & 0.16 & -0.16\end{array}$

$\begin{array}{lll}-0.04 & 0.16 & -0.22\end{array}$

$\begin{array}{lll}-0.32 & 0.16 & -1.98 *\end{array}$

1.12

$35.6 \%$

Slope $(M=1.61, S E=0.35)$

$B \quad S E \quad t$

$\begin{array}{lll}-0.26 & 0.19 & -1.38\end{array}$

$\begin{array}{lll}0.47 & 0.17 & 2.74 *\end{array}$

$\begin{array}{lll}0.07 & 0.19 & 0.36\end{array}$

$\begin{array}{lll}0.06 & 0.18 & 0.93\end{array}$

$\begin{array}{lll}-0.07 & 0.21 & -0.34\end{array}$

$\begin{array}{lll}-0.03 & 0.16 & -0.21\end{array}$

$\begin{array}{lll}0.01 & 0.17 & 0.06\end{array}$

$\begin{array}{lll}-0.18 & 0.19 & -0.96\end{array}$

1.68

$19.7 \%$

$* p<0.05, * * p<0.01, * * * p<0.001$

\section{Discussion}

The main aim of the present study was to examine the interaction of marital conflict and RSA activity on the development of externalizing problems when controlling for internalizing problems among Chinese adolescents. Consistent with the hypothesis, the present study found that marital conflict and RSA reactivity to the speech task interact to predict the initial level 


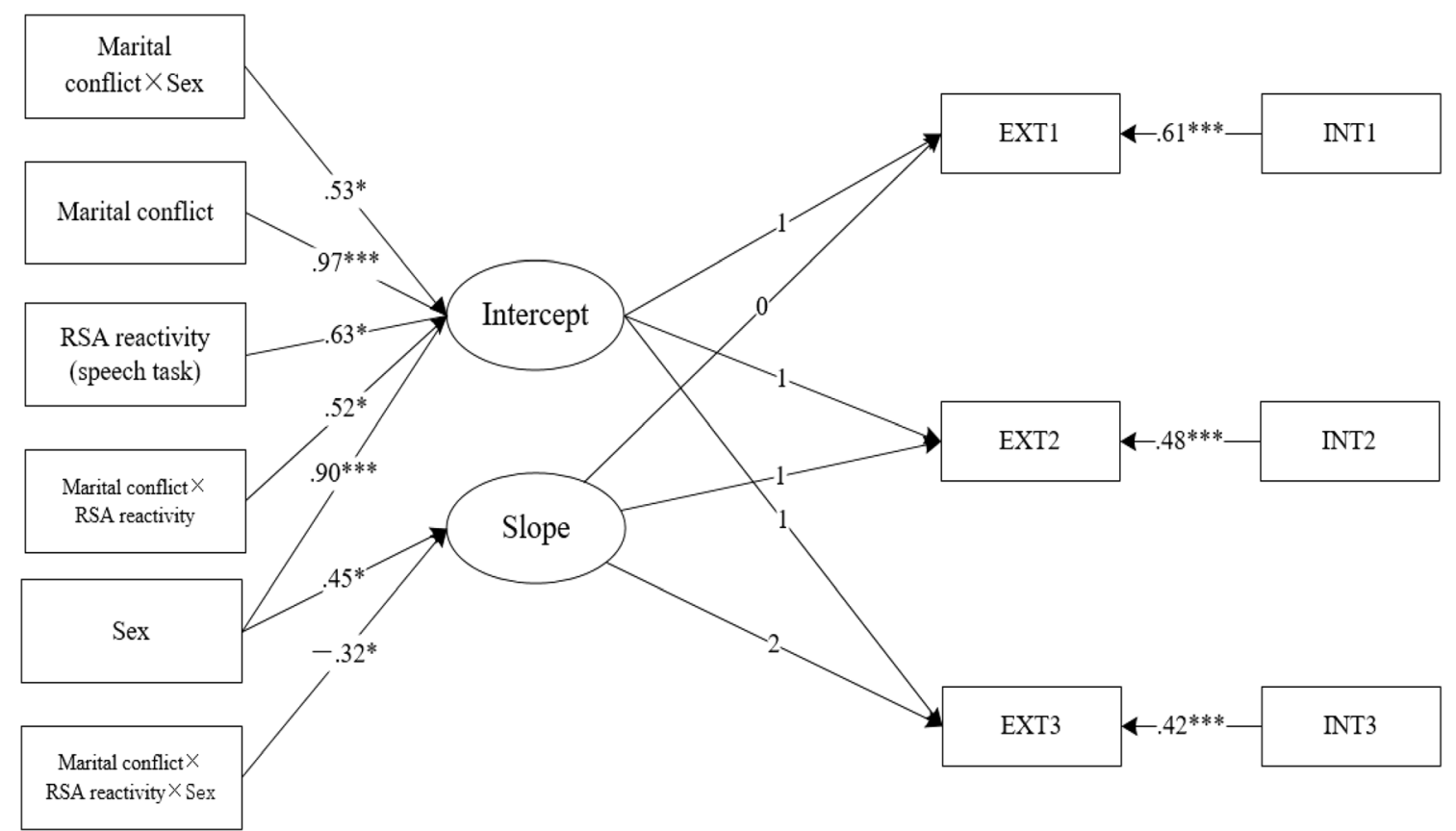

Fig. 2 Structural equation model examining interaction role of marital conflict and RSA reactivity (speech task) on adolescents' externalizing problems. Parameter estimates for the structural paths are unstandardized

path. EXT $=$ externalizing problems, INT $=$ internalizing problems, RSA $=$ respiratory sinus arrhythmia. Note: $* p<0.05, * * * p<0.001$

of externalizing problems when controlling for internalizing problems. Moreover, the present study found that marital conflict, RSA reactivity to the speech task, and adolescents' sex interactively predicted the development of externalizing problems when controlling for internalizing problems. However, the interactions were not significant when internalizing problems were not controlling for.

As expected, the present study suggested that the contribution of marital conflict and RSA activity to externalizing problems might be affected by the covariance of externalizing and internalizing problems. Comorbidity or covariation of externalizing problems and internalizing problems are at a greater rate than expected by chance during childhood and adolescence (Achenbach et al. 2016; Angold and Costello 1993). Thus, it has been suggested that for better understanding of

the etiology and development of externalizing problems, the covariation of externalizing and internalizing problems should be considered. Consistent with prior studies (e.g., Calkins et al. 2007), the present study further demonstrated that different patterns of externalizing problems were related to differential patterns of RSA reactivity to environmental challenges. When controlling for internalizing problems, which was aimed to remove the impact of the covariance of externalizing and internalizing problems, the moderating effect of RSA reactivity to the speech task on the association between marital conflict and externalizing problems was significant. Therefore, it is necessary to partial out the impact of covariation of externalizing problems and internalizing problems when examining the associations among RSA activity, marital conflict and externalizing problems.

Fig. 3 The simple slope of marital conflict on the intercept of externalizing problems in adolescents. Dotted lines represent upper and lower 95\% confidence intervals of the simple slope. The shaded areas represent the region of significance. RSA = respiratory sinus arrhythmia

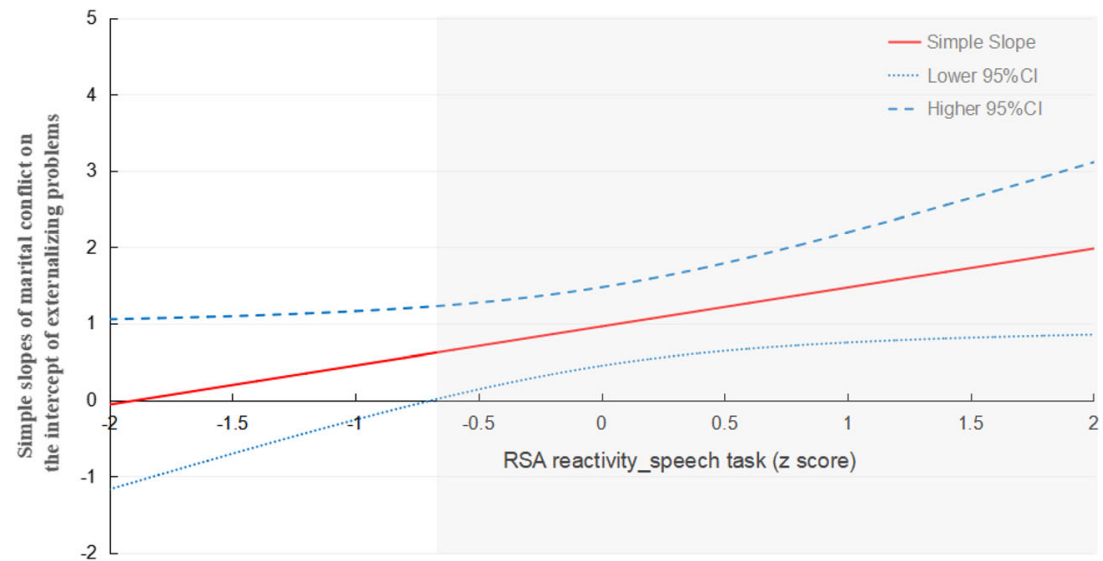




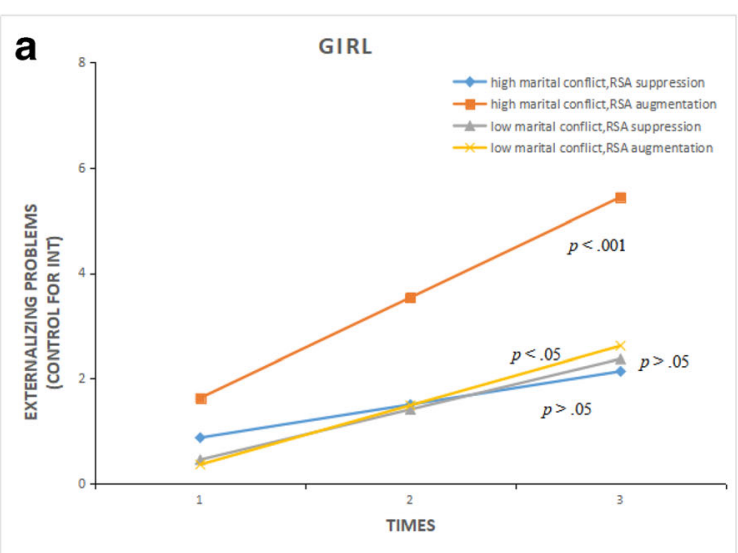

Fig. 4 a-b Change in externalizing problems (control for internalizing problems) over time for prototypical boys and girls in the population at high and low values $(M=1 \pm S D)$ of RSA reactivity (speech task) and

The present study found that marital conflict, RSA reactivity to the speech task, and adolescents' sex interactively predict the development of externalizing problems over time when controlling for internalizing problems. Specifically, lower RSA suppression or RSA augmentation in response to the speech task combined with marital conflict contributed to both girls' and boys' high level of concurrent and prospective externalizing problems. While, in the context of marital conflict, girls with greater RSA suppression in response to the speech task reported low and stable externalizing problems. For boys with the same pattern, their externalizing problems slightly increased in the context of low marital conflict. These findings were consistent with the perspective of the diathesisstress model (Monroe and Simons 1991) that dual risks, including marital conflict and RSA augmentation in response to stress, had the worst impact on adolescents' developmental outcomes, and such influence was demonstrated to be persistent (i.e., the current dual risks may increase the likelihood of future externalizing problems). Resilience characteristics, such as greater RSA suppression in response to stress, attenuated the negative impact of the adverse family environment on adolescents' behavioral outcomes. As mentioned above, greater RSA suppression in response to stress reflected an individual's ability to modulate their physiological state sufficiently and flexibly. Thus, girls with greater RSA suppression in response to the speech task were more likely to form an organized response and regulate emotion and behavior adaptively; therefore, over time, girls with greater RSA suppression could experience fewer detrimental effects of chronic physiological arousal induced by frequent marital conflict (Calkins et al. 2007; El-Sheikh and Erath 2011; Graziano and Derefink 2013). In addition, contrary to the hypotheses, the present study found that the protective effect of RSA suppression in response to the speech task was stronger among girls than boys. The less protective effect of RSA suppression among boys might be partly due to the significant increase in

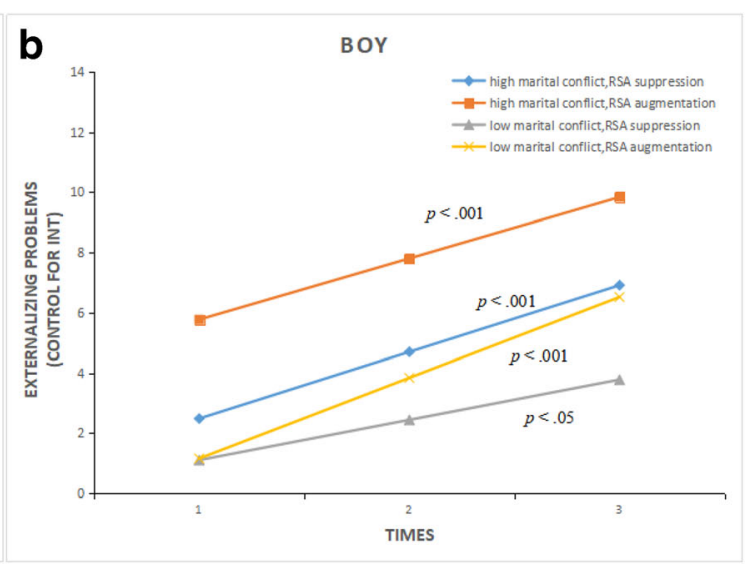

marital conflict. The three-way interaction (RSA reactivity, Marital Conflict and Sex $)$ predicts the slope $(p<0.05) . \mathrm{RSA}=$ respiratory sinus arrhythmia

externalizing problems than that of girls during early adolescence (Ullsperger and Nikolas 2017). This finding further suggested that the same physiological response (RSA suppression) might have different protective effects on girls' and boys' externalizing problems in risk environments (ElSheikh et al. 2011; Graziano and Derefink 2013).

The present study did not find that RSA reactivity to the mental arithmetic task interacts with marital conflict to predict the development of externalizing problems, regardless of whether internalizing problems were controlled for. As prior studies suggested, the types of stressor tasks used to evoke RSA reactivity might affect the moderating effect of RSA reactivity on the relationship between family environment and psychological adaptation among children and adolescents (Cui et al. 2015; Obradović et al. 2011). Under low-challenge context, such as simple mental arithmetic tasks, RSA suppression might not represent adaptive physiological regulation (Davis et al. 2016; Hastings et al. 2008), which may account for the null finding.

The present study did not find that baseline RSA had direct and moderating effects on externalizing problems regardless of whether internalizing problems were controlled for. This aligned with a previous study that showed a nonsignificant relationship between baseline RSA and children's concurrent pure externalizing problems (Calkins et al. 2007). As previous studies have proposed that the pattern of the relationships between externalizing problems and baseline RSA is less consistent for RSA suppression (Eisenberg et al. 2012), the findings further suggested that baseline RSA might not be a robust correlate of externalizing problems. It was well known that one of the most replicated biological markers of externalizing problems in adolescence was a low resting heart rate, which was influenced by both the sympathetic nervous system and PNS (Ortiz and Raine 2004; Raine 2002). Thus, the physiological vulnerability of externalizing problems might be regarded as a complex co-functioning of the resting 
Table 3 Estimates for predictors of externalizing problems trajectory (not control for internalizing problems)

Model 1

Main effects

Sex

Marital conflict

Baseline RSA

2-way interactions

Marital conflict $\times$ Baseline RSA

Sex $\times$ Marital conflict

Sex $\times$ Baseline RSA

3-way interaction

Marital conflict $\times$ Baseline RSA $\times$ Sex

Residual variance

$R^{2}$

Model 2

Baseline RSA

Main effects

Sex

Marital conflict

RSA reactivity (speech task)

2-way interactions

Marital conflict $\times$ RSA reactivity (speech task)

Sex $\times$ Marital conflict

Sex $\times$ RSA reactivity (speech task)

3-way interaction

Marital conflict $\times$ RSA reactivity (speech task) $\times$ Sex

Residual variance

$R^{2}$

Model 3

Baseline RSA

Main effects

Sex

Marital conflict

RSA reactivity (mental arithmetic task)

2-way interactions

Marital conflict $\times$ RSA reactivity (mental arithmetic task)

Sex $\times$ Marital conflict

Sex $\times$ RSA reactivity (mental arithmetic task)

3-way interaction

Marital conflict $\times$ RSA reactivity (mental arithmetic task) $\times$ Sex

Residual variance

$R^{2}$

$* p<0.05, * * p<0.01, * * * p<0.001$

sympathetic nervous system and PNS. However, this speculation must be further explored in the future.

To our knowledge, this was the first longitudinal study to examine the interaction between marital conflict and RSA
Intercept $(M=9.04, S E=0.37)$

$S E$

$t$

0.62

2.01

\subsection{7}

1.68

$5.27 * * *$

0.21

0.38

$-0.54$

0.77

0.37

$-1.44$

$2.05 *$

$-0.48$

$-1.29$

$-0.05$

0.36

$-0.13$

22.04

$17.8 \%$

Intercept $(M=9.03, S E=0.36)$

0.53

0.61

2.07

0.91

0.45

0.80

1.15

$$
S E
$$

0.41

$t$

3

\subsection{6}

0.38

0.41

1.68

$5.52 * * *$

$2.20 *$

0.36

0.37

0.37

\subsection{0}

$2.16^{*}$

$3.12 * *$

0.37

1.06

19.24

$25.1 \%$

Intercept $(M=9.01, S E=0.37)$

$B$
0.54

0.58

2.02

0.96

0.64

0.73

0.72

0.60

20.1

$23.4 \%$

$\begin{array}{lll}0.37 & 0.37\end{array}$

$0.38 \quad 1.89$

$0.44 \quad 1.37$
Slope $(M=1.28, S E=0.23)$
$B \quad S E \quad t$

$\begin{array}{lll}0.34 & 0.22 & 1.50 \\ 0.03 & 0.24 & 0.13 \\ -0.32 & 0.23 & -1.40\end{array}$

$\begin{array}{lll}-0.07 & 0.23 & -0.26\end{array}$

$\begin{array}{lll}-0.19 & 0.22 \quad-0.86\end{array}$

$\begin{array}{lll}0.09 & 0.22 & 0.41\end{array}$

$\begin{array}{lll}0.08 & 0.23 & 0.33\end{array}$

7.31

$4.5 \%$

Slope $(M=1.28, S E=0.23)$

$B \quad S E \quad t$

$\begin{array}{lll}-0.21 & 0.25 & -0.83\end{array}$

$\begin{array}{lll}0.31 & 0.22 & 1.41\end{array}$

$\begin{array}{lll}0.04 & 0.24 & 0.18\end{array}$

$\begin{array}{lll}0.28 & 0.25 & 1.10\end{array}$

$\begin{array}{lll}0.03 & 0.23 & 0.12\end{array}$

$\begin{array}{lll}-0.21 & 0.23 & -1.02\end{array}$

$\begin{array}{lll}-0.23 & 0.22 & -0.95\end{array}$

$\begin{array}{lll}-0.36 & 0.23 & -1.54\end{array}$

6.32

$8.5 \%$

Slope $(M=1.30, S E=0.23)$

$B \quad S E \quad t$

$\begin{array}{lll}-0.35 & 0.25 & -1.39\end{array}$

$\begin{array}{llllll} & S E & t & B & S E & t \\ 0.54 & 0.41 & 1.34 & -0.35 & 0.25 & -1.39\end{array}$

$\begin{array}{llllll}58 & 0.37 & 1.57 & 0.32 & 0.23 & 1.43\end{array}$

$\begin{array}{lllll}0.38 & 5.35 * * * & 0.04 & 0.24 & 0.18\end{array}$

$\begin{array}{lllll}0.42 & 2.29 * & -0.07 & 0.27 & -0.25\end{array}$

$\begin{array}{llllll}0.45 & 1.43 & 0.05 & 0.27 & 0.17\end{array}$

$-0.20 \quad 0.22$

$-0.90$

$-0.14 \quad 0.23$

$-0.64$

-0.18
6.92

0.28

$-0.65$

$5.5 \%$ activity on externalizing problems when controlling for internalizing problems among Chinese adolescents. Consistent with the findings in the Western context, studies in Chinese culture, which prefered avoidant, subtle, and indirect 
approaches to conflict resolution (Bond and Wang 1983), also found that marital conflict is associated with adolescents' externalizing behavior. Furthermore, adolescents' PNS regulation could moderate this association. Therefore, interventions in Western contextual studies that focusing on reducing marital conflict and improving children's and adolescents' PNS function could also alleviate Chinese adolescents' externalizing problems.

\section{Limitations}

Several limitations of the present study should be considered. First, low marital conflict did not necessarily indicate a supportive and nurturing environment; further studies should explore whether issues exist in positive family environments. Second, externalizing and internalizing problems and marital conflict scores were obtained through adolescents' self-reports, and more comprehensive, multi-informant assessments should be included in future research to fully evaluate the effect of family environments and RSA activity on adolescents.

\section{Conclusions and Implications}

Despite these limitations, this study provided an important step toward further understanding how marital conflict and RSA activity may contribute to adjustment problems in adolescence. Consistent with the polyvagal theory (Porges 1995, 2007), this finding suggested that adaptive PNS regulation may provide the resources necessary for meeting environmental demands and may be protective against adverse family environments. However, maladaptive PNS evoked in the family conflict context may extend to multiple domains of adolescents' adjustment, including the development of externalizing problems over time. Importantly, the present study highlighted the importance of considering the covariation of externalizing and internalizing problems when trying to understand the interaction effects between PNS functioning and family stressors in the prediction of externalizing problems.

Funding This research was supported by National Science Foundation Grant (31671152) of China, and the Fundamental Research Funds for the Central Universities (2018TS085, GK201903107).

\section{Compliance with Ethical Standards}

Conflict of Interest Authors Yuan Peng, Xiaohui Yang and Zhenhong Wang declare that they have no conflict of interest.

Ethical Approval All procedures were in accordance with the ethical standards of the institutional research committee and with the 1964
Helsinki declaration and its later amendments or comparable ethical standards.

Informed Consent Informed consent was obtained from all individual participants included in the study.

Open Access This article is licensed under a Creative Commons Attribution 4.0 International License, which permits use, sharing, adaptation, distribution and reproduction in any medium or format, as long as you give appropriate credit to the original author(s) and the source, provide a link to the Creative Commons licence, and indicate if changes were made. The images or other third party material in this article are included in the article's Creative Commons licence, unless indicated otherwise in a credit line to the material. If material is not included in the article's Creative Commons licence and your intended use is not permitted by statutory regulation or exceeds the permitted use, you will need to obtain permission directly from the copyright holder. To view a copy of this licence, visit http://creativecommons.org/licenses/by/4.0/.

\section{References}

Achenbach, T.M., \& Rescorla, L.A. (2001). Manual for the ASEBA School-Age Forms and Profiles. Burlington, VT: University of Vermont, Research Center for Children, Youth and Families.

Achenbach, T. M., Ivanova, M. Y., Rescorla, L. A., Turner, L. V., \& Althoff, R. R. (2016). Internalizing/externalizing problems: review and recommendations for clinical and research applications. Journal of the American Academy of Child and Adolescent Psychiatry, 55(8), 647-656. https://doi.org/10.1016/j.jaac.2016.05.012.

Angold, A., \& Costello, E. J. (1993). Depressive comorbidity in children and adolescents: Empirical, theoretical, and methodological issues. American Journal of Psychiatry, 150, 1779-1791. https://doi.org/ 10.1176/ajp.150.12.1779.

Bauer, D. J., \& Curran, P. J. (2005). Probing interactions in fixed and multilevel regression: inferential and graphical techniques. Multivariate Behavioral Research, 40, 373-400. https://doi.org/10. 1207/s15327906mbr4003_5.

Beauchaine, T. P., Gatzke-Kopp, L., \& Mead, H. K. (2007). Polyvagal theory and developmental psychopathology: emotion dysregulation and conduct problems from preschool to adolescence. Biological Psychology, 74(2), 174-184. https://doi.org/10.1016/j.biopsycho. 2005.08.008.

Belsky, J., \& Pluess, M. (2009). Beyond diathesis stress: differential susceptibility to environmental influences. Psychological Bulletin, 135(6), 885-908. https://doi.org/10.1037/a0017376.

Blood, J. D., Wu, J., Chaplin, T. M., Hommer, R., Vazquez, L., Rutherford, H. J. V., \& Crowley, M. J. (2015). The variable heart: High frequency and very low frequency correlates of depressive symptoms in children and adolescents. Journal of Affective Disorders, 186, 119-126. https://doi.org/10.1016/j.jad.2015.06. 057.

Bond, M. H., \& Wang, S. H. (1983). Aggressive behavior in Chinese society: the problem of maintaining order and harmony. In A. P. Goldstein \& M. Segall (Eds.), Global perspectives on aggression (pp. 58-74).

Bosch, N. M., Riese, H., Ormel, J., Verhulst, F., \& Oldehinkel, A. J. (2009). Stressful life events and depressive symptoms in young adolescents: Modulation by respiratory sinus arrhythmia? The TRAILS study. Biological Psychology, 81(1), 40-47. https://doi. org/10.1016/j.biopsycho.2009.01.005.

Calkins, S. D., Graziano, P. A., \& Keane, S. P. (2007). Cardiac vagal regulation differentiates among children at risk for behavior 
problems. Biological Psychology, 74, 144-153. https://doi.org/10. 1016/j.biopsycho.2006.09.005.

Chen, F. F., West, S. G., \& Sousa, K. H. (2006). A comparison of bifactor and second-order models of quality of life. Multivariate Behavioral Research, 41, 189-225. https://doi.org/10.1207/ s15327906mbr4102 5 .

Chi, L. P., \& Xin, Z. Q. (2003). The revision of children's perception of marital conflict scale. Chinese Mental Health Journal, 17(8), 554 556.

Cicchetti, D. (2008). Amultiple-levels-of-analysis perspective on research in development and psychopathology. In T. Beauchaine \& S. Hinshaw (Eds.), Child and adolescent psychopathology (pp. 2757). Hoboken: Wiley.

Cui, L., Morris, A. S., Harrist, A. W., Larzelere, R. E., Criss, M. M., \& Houltberg, B. J. (2015). Adolescent RSA responses during an anger discussion task: relations to emotion regulation and adjustment. Emotion, 15(3), 360-372. https://doi.org/10.1037/emo0000040.

Cummings, E. M., \& Davies, P. T. (2010). Marital conflict and children: an emotional security perspective. Guilford Press.

Davis, M., Suveg, C., Whitehead, M., Jones, A. M., \& Shaffer, A. (2016). Preschoolers' psychophysiological responses to mood induction tasks moderate the intergenerational transmission of internalizing problems. Biological Psychology, 159-169. https://doi.org/10. 1016/j.biopsycho.2016.03.015

Diamond, L. M., Fagundes, C. P., \& Cribbet, M. R. (2012). Individual differences in adolescents' sympathetic and parasympathetic functioning moderate associations between family environment and psychosocial adjustment. Developmental Psychology, 48(4), 918-931. https://doi.org/10.1037/a0026901.

Dickerson, S. S., \& Kemeny, M. E. (2004). Acute stressors and cortisol responses: a theoretical integration and synthesis of laboratory research. Psychological Bulletin, 130, 355-391. https://doi.org/10. 1037/0033-2909.130.3.355.

Dietrich, A., Riese, H., Sondeijker, F. E., Greaves-Lord, K., Ormel, J., Neeleman, J., \& Rosmalen, J. G. (2007). Externalizing and internalizing problems in relation to autonomic function: a population-based study in preadolescents. Journal of the American Academy of Child and Adolescent Psychiatry, 6(3), 378-386. https://doi.org/10.1097/ CHI.0b013e31802b91ea

Dietrich, A., Greaves-Lord, K., Bosch, N. M., Oldehinkel, A. J., Minderaa, R.B., Hoekstra, P.J., \& Althaus, M. (2011). Reduced cardiac autonomic flexibility associated with medically unexplained somatic complaints in the context of internalizing symptoms in a preadolescent population sample: The TRAILS study. Psychotherapy and Psychosomatics , 80(1), 62-64. https://doi.org/ 10.1159/000318781.

Eisenberg, N., Sulik, M. J., Spinrad, T. L., Edwards, A., Eggum, N. D., Liew, J., Sallquist, J., Popp, T. K., Smith, C. L., \& Hart, D. (2012). Differential susceptibility and the early development of aggression: interactive effects of respiratory sinus arrhythmia and environmental quality. Developmental Psychology, 48(3), 755-768. https://doi.org/ 10.1037/a0026518.

El-Sheikh, M., \& Erath, S. A. (2011). Family conflict, autonomic nervous system functioning, and child adaptation: state of the science and future directions. Development and Psychopathology, 23(2), 703721. https://doi.org/10.1017/S0954579411000034.

El-Sheikh, M., \& Whitson, S. A. (2006). Longitudinal relations between marital conflict and child adjustment: vagal regulation as a protective factor. Journal of Family Psychology, 20(1), 30-39. https://doi. org/10.1037/0893-3200.20.1.30.

El-Sheikh, M., Harger, J., \& Whitson, S. (2001). Exposure to parental conflict and children's adjustment and physical health: the moderating role of vagal tone. Child Development, 72(6), 1617-1636. https://doi.org/10.1111/1467-8624.00369.

El-Sheikh, M., Hinnant, J. B., \& Erath, S. (2011). Developmental trajectories of delinquency symptoms in childhood: the role of marital conflict and autonomic nervous system activity. Journal of Abnormal Psychology, 120(1), 16-32. https://doi.org/10.1037/ a0020626.

Graziano, P., \& Derefink, K. (2013). Cardiac vagal control and children's adaptive functioning: a meta-analysis. Biological Psychology, 94, 22-37. https://doi.org/10.1016/j.biopsycho.2013.04.011.

Grych, J. H., \& Fincham, F. D. (1990). Marital conflict and children's adjustment: a cognitive-contextual framework. Psychology Bullutin, 108, 267-290. https://doi.org/10.1037/0033-2909.108.2.267.

Grych, J. H., \& Fincham, F. D. (2001). Interparental conflict and child development : theory, research, and applications. Cambridge: Cambridge University Press.

Hastings, P. D., Nuselovici, J. N., Utendale, W. T., Coutya, J., McShane, K. E., \& Sullivan, C. (2008). Applying the polyvagal theory to children's emotion regulation: social context, socialization, and adjustment. Biological Psychology, 79, 299-306. https://doi.org/10. 1016/j.biopsycho.2008.07.005.

Hu, L., \& Bentler, P. M. (1999). Cutoff criteria for fit indexes in covariance structure analysis: conventional criteria versus new alternatives. Structural Equation Modeling, 6(1), 1-55. https://doi.org/10. 1080/10705519909540118.

Katz, L. F., \& Gottman, J. M. (1997). Buffering children from marital conflict and dissolution. Journal of Clinical Child Psychology, 26(2), 157-171. https://doi.org/10.1207/s15374424jccp2602 4.

Keiley, M. K., Lofthouse, N., Bates, J. E., Dodge, K. A., \& Pettit, G. S. (2003). Differential risks of covarying and pure components in mother and teacher reports of externalizing and internalizing behavior across ages 5 to 14. Journal of Abnormal Child Psychology, 31(3), 267-283. https://doi.org/10.1023/A:1023277413027.

Koenig, J., Kemp, A. H., Beauchaine, T. P., Thayer, J. F., \& Kaess, M. (2016). Depression and resting state heart rate variability in children and adolescents - a systematic review and meta-analysis. Clinical Psychology Review, 46, 136-150. https://doi.org/10.1016/j.cpr. 2016.04.013.

Lang, P. J., Bradley, M. M., \& Cuthbert, B. N. (2005). International affective picture system (IAPS): Affective rating of pictures and instruction manual. Gainesville, FL: University of Florida Center for Research in Psychophysiology.

Lilienfeld, S. O. (2003). Comorbidity between and within childhood externalizing and internalizing disorders: reflections and directions. Journal of Abnormal Child Psychology, 31(3), 285-291. https:// doi.org/10.1023/A:1023229529866.

Lü, W., Wang, Z. H., \& Hughes, B. M. (2016). Openness and physiological responses to recurrent social stress. International Journal of Psychophysiology, 106, 135-140. https://doi.org/10.1016/j. ijpsycho.2016.05.004.

Martinstorey, A., Temcheff, C. E., Dery, M., Poirier, M., Verlaan, P., \& Lemelin, J. (2018). Trajectories of internalizing problems during the transition to adolescence in children with and without conduct problems. International Journal of Behavioral Development, 42(5), 464-473. https://doi.org/10.1177/0165025417730681.

McCormick, C. M., Mathews, I. Z., Thomas, C., \& Waters, P. (2010). Investigations of HPA function and the enduring consequences of stressors in adolescence in animal models. Brain and Cognition, 72, 73-85. https://doi.org/10.1016/j.bandc.2009.06.003.

Mcquade, J. D., \& Breaux, R. P. (2017). Parent emotion socialization and pre-adolescent's social and emotional adjustment: moderating effects of autonomic nervous system reactivity. Biological Psychology, 130, 67-76. https://doi.org/10.1016/j.biopsycho.2017. 10.007 .

Monroe, S. M., \& Simons, A. D. (1991). Diathesis-stress theories in the context of life stress research: implication for the depressive disorders. Psychological Bulletin, 110(3), 406-425. https://doi.org/10. 1037/0033-2909.110.3.406.

Obradović, J., Bush, N. R., \& Boyce, W. T. (2011). The interactive effect of marital conflict and stress reactivity on externalizing and 
internalizing symptoms: the role of laboratory stressors. Development and Psychopathology, 23(1), 101-114. https://doi. org/10.1017/S0954579410000672.

Ortiz, J., \& Raine, A. (2004). Heart rate level and antisocial behavior in children and adolescents: a meta-analysis. Journal of American Academy of Child and Adolescent Psychiatry, 43, 154-162. https://doi.org/10.1097/00004583-200402000-00010.

Palatini, P., Bratti, P., Palomba, D., Bonso, E., Saladini, F., Benetti, E., et al. (2011). Bp reactivity to public speaking in stage 1 hypertension: influence of different task scenarios. Blood Pressure, 20(5), 290-295. https://doi.org/10.3109/08037051.2011.566011.

Pang, K. C., \& Beauchaine, T. P. (2013). Longitudinal patterns of autonomic nervous system responding to emotion evocation among children with conduct problems and/or depression. Developmental Psychobiology, 55(7), 698-706. https://doi.org/10.1002/dev.21065.

Porges, S. W. (1995). Orienting in a defensive world: Mammalian modifications of our evolutionary heritage. a polyvagal theory. Psychophysiology, 32(4), 301-318. https://doi.org/10.1111/j.14698986.1995.tb01213.x.

Porges, S. W. (2007). The polyvagal perspective. Biological Psychology, 74(2), 116-143. https://doi.org/10.1016/j.biopsycho.2006.06.009.

Raine, A. (2002). Biosocial studies of antisocial and violent behavior in children and adults: a review. Journal of Abnormal Child Psychology, 30, 311-326. https://doi.org/10.1023/A: 1015754122318.

Schafer, J. L., \& Graham, J. W. (2002). Missing data: our view of the state of the art. Psychological Methods, 7, 147-177. https://doi.org/10. 1037/1082-989X.7.2.147.

Shader, T. M., Gatzkekopp, L. M., Crowell, S. E., Jamila, R. M., Thayer, J. F., Vasey, M. W., et al. (2018). Quantifying respiratory sinus arrhythmia: effects of misspecifying breathing frequencies across development. Development and Psychopathology, 30(1), 351-366. https://doi.org/10.1017/S0954579417000669.

Singer, J. D., \& Willett, J. B. (2003). Applied longitudinal data analysis: modeling change and event occurrence. Oxford: Oxford University Press.
Tarvainen, M. P., Ranta-aho, P. O., \& Karjalainen, P. A. (2002). An advanced detrending method with application to HRV analysis. IEEE Transactions on Bio-medical Engineering, 49(2), 172-175. https://doi.org/10.1109/10.979357.

Thayer, J. F., \& Lane, R. D. (2000). A model of neurovisceral integration in emotion regulation and dysregulation. Journal of Affective Disorders, 61(3), 201-216. https://doi.org/10.1016/S01650327(00)00338-4.

Tu, K. M., Erath, S. A., \& El-Sheikh, M. (2016). Coping responses moderate prospective associations between marital conflict and youth adjustment. Journal of Family Psychology, 30(5), 523-532. https://doi.org/10.1037/fam0000169.

Ullsperger, J. M., \& Nikolas, M. A. (2017). A meta-analytic review of the association between pubertal timing and psychopathology in adolescence: are there sex differences in risk? Psychological Bulletin, 143(9), 903-938. https://doi.org/10.1037/bul0000106.

Wang, R. C., Wang, M. C., Gao, Y. D., Jiang, Y. L., Zhang, X. C., \& Yao, S. Q. (2016). Reliability and validity of the Chinese version of Achenbach Youth Self-Report (2001 Version). Chinese Journal of Clinical Psychology, 21(6), 977-980. https://doi.org/10.16128/j. cnki.1005-3611.2013.06.036.

Wang, F. L., Eisenberg, N., Valiente, C., \& Spinrad, T. L. (2016). Role of temperament in early adolescent pure and co-occurring internalizing and externalizing problems using a bifactor model: moderation by parenting and gender. Development and Psychopathology, 28(4), 1487-1504. https://doi.org/10.1017/s0954579415001224.

Whitson, S. M., \& El-Sheikh, M. (2003). Moderators of family conflict and children's adjustment and health. Journal of Emotional Abuse, 3(1), 47-73. https://doi.org/10.1300/J135v03n01_03.

Xu, Y., Raine, A., Yu, L., \& Krieg, A. (2014). Resting heart rate, vagal tone, and reactive and proactive aggression in Chinese children. Journal of Abnormal Child Psychology, 42, 501-514. https://doi. org/10.1007/s10802-013-9792-2.

Publisher's Note Springer Nature remains neutral with regard to jurisdictional claims in published maps and institutional affiliations. 